and $70 \%$ of such attacks were executed by Kurdish women in Turkey (Pope, 2005). There is also a high proportion of women suicide bombers in the Tamil Tigers $(30 \%)$. al Qa'ida, which associates itself with Islamic fundamentalism, never used female suicide attackers from its formation in 1993 until the tragic attack in Jordan in 2005.

In general, women are at a lower risk of suicide than men and a protective effect of child-bearing in terms of suicide risk has been postulated (Catalan, 2000). This does not appear to apply to female suicide bombers or to some countries and cultures in which gender representation in suicide is reversed. A higher female:male suicide ratio is not unique to China. The significantly higher rate of female suicide observed outside China is not an 'ecological fallacy' but a sad reality.

Catalan, J. (2000) Sexuality, reproductive cycle and suicidal behaviour. In International Handbook of Suicide and Attempted Suicide (eds K. Hawton \& K. van Heeringen), pp. 294-307. John Wiley \& Sons.

Cheng, A. T. A. \& Lee, C. (2000) Suicide in Asia and the Far East. In International Handbook of Suicide and Attempted Suicide (eds K. Hawton \& K. van Heeringen), pp. 29-48. John Wiley \& Sons.

Pope, R. A. (2005) Dying to Win. The Strategic Logic of Suicide Terrorism, pp. 203-216. Random House.

Soni Raleigh, V., Bulusu, L. \& Balarajan, R. (1990)

Suicides among immigrants from the Indian

subcontinent. British Journal of Psychiatry, 156, 46-50.

Tadros, G. \& Salib, E. (2006) Elderly suicide in primary care. International Journal of Geriatric Psychiatry, doi: 10.1002/gps.1734 (Epub ahead of print)

Yip, P. S. F. \& Liu, K. Y. (2006) The ecological fallacy and the gender ratio of suicide in China. British Journal of Psychiatry, 189, 465-466.

E. Salib Peasley Cross Hospital, St Helens, and Honorary Senior Lecturer, Liverpool University Email: esalib@hotmail.com

G. Tadros Birmingham and Solihull Mental Health Trust, Birmingham, UK

doi: 10.I192/bjp.190.3.273a

Authors' reply Salib \& Tadros highlight the important issue of high female suicide rates among Indian migrants and the use of female suicide bombers. Like the high suicide rates among young females in rural China (Yip \& Liu, 2006), these deviations from the general pattern should not be discounted as mere exceptions but should be considered as representative of the distressing situations faced by some women in Asia.
We also believe that socio-economic deprivation and poor social support - the 'sad reality' - faced by young women in rural China are underlying causes of the high suicide rates. Like the young married Indian women in Britain, there is some indication that young married women in rural China might be at high risk (Pearson et al, 2002). This reminds us that the lives of married women differ greatly across regions, countries, cultures and economies, and there is a need to avoid oversimplification when describing suicide in different countries; one size does not fit all.

Over $60 \%$ of the world's suicides occur in Asian countries where low male:female ratios for suicide are common (Yip et al, 2000). Although the official male:female ratio for suicide in India was still greater than 1 (1.2:1 in 2002), the ratio was 0.8 among those aged 14 or below (World Health Organization, 2006). However, unlike China (Yip \& Liu, 2006), the small size of this population subgroup meant that the national male:female ratio remained greater than 1. (This is the essence of our ecological fallacy argument.) In addition to specific social factors, the similarity in the methods of suicide used by males and females, together with the poor access to medical facilities, might explain the low male:female ratio in India and China. Restricting access to pesticides will prevent many suicides in Asia. In the long term improving economic and educational opportunities, especially for rural women in deprived areas, raising awareness of depression and better treatment will be pivotal for preventing suicides.

Pearson, V., Phillips, M. R., He, F., et al (2002) Attempted suicide among young rural women in the People's Republic of China: possibilities for prevention Suicide and Life Threatening Behavior, 32, 359-369.

Yip, P. S., Callanan, C. \& Yuen, H. P. (2000) Urban/ rural and gender differentials in suicide rates: east and west. Journal of Affective Disorders, 57, 99-106.

World Health Organization (2006) Country Report on Suicide (India).World Health Organization. http: / / www.who.int/entity/mental_health/media/indi.pdf

K. Y. Liu Nuffield College, University of Oxford, Oxford, UK

P. S. F. Yip Hong Kong Jockey Club Centre for Suicide Research and Prevention, University of Hong Kong, Hong Kong. Email: sfpyip@hku.hk doi: 10.1192/bjp.190.3.274

\section{Self-poisoning with pesticides in India}

Bertolote et al (2006) report the global response to deaths from pesticide poisoning. Suicide rates in southern India have been reported to be high (Joseph et al, 2003; Aaron et al, 2004; Abraham et al, 2005; Prasad et al, 2006), with 1741 suicides documented in a population of about 100000 from 1986 to 2005. Hanging (804 of $1741,46.2 \%)$ and poisoning (746 of $1741,42.8 \%$ ) were the methods most commonly employed. Although people under 40 years tended to use poisons, older people tended to choose hanging $\left(\chi^{2}=36.71\right.$, d.f. $\left.=4, P<0.001\right)$. Significantly more males (465 of $984,47.3 \%$ ) than females (281 of $757,37.1 \%) \quad\left(\chi^{2}=17.6\right.$, d.f. $=1, P<0.001)$ chose death by poisoning. There was no significant change in the overall rate of suicide or the method employed during the period. Detailed analysis of the data from 2001 to 2005 revealed that only $68 \%$ of the fatal episodes of self-poisoning were a result of ingestion of pesticides.

Self-poisoning with pesticides is a significant public health problem in low- and middle-income countries. The majority of such poisoning occurs in rural agrarian households. Some suggestions to reduce such deaths are currently difficult to implement. Enforcing the hazardous chemicals and wastes conventions to restrict and control the sale and use of pesticides in such regions is no small task and requires major political, administrative, financial and social commitment. Given the many competing demands on limited governmental resources in low- and middle-income countries, such protocols are difficult to implement. In addition, the improved recognition and treatment of mental illness may not have a significant impact on the overall suicide rate as many people in the low- and middle-income countries who die by suicide do not have severe mental illness. Rather, the majority of such attempts are impulsive and follow stressful life events. Although reducing accessibility to pesticides will decrease such impulsive attempts and consequent deaths, social, economic and cultural factors must also be addressed to make a real difference. Thus, although the World Health Organization's intersectoral global initiative is a step in the right direction, it is imperative that practical issues related to its implementation are discussed. It is necessary to consider 
strategies to encourage governments to set up suicide prevention programmes to reduce suicide rates in populations as a whole rather than method- and site-specific rates.

Suicide in low- and middle-income countries is not only a medical and public health problem but is also related to economics and culture. A coordinated and a comprehensive response is needed to make any impact.
Aaron, R., Joseph, A., Abraham, S., et al (2004)

Suicides in young people in rural southern India. Lancet, 363, $1117-1118$.

Abraham, V. J., Abraham, S. \& Jacob, K. S. (2005) Suicide in the elderly in Kaniyambadi block, Tamil Nadu, South India. International Journal of Geriatric Psychiatry, 20, 953-955.

Bertolote, J. M., Fleischmann, A., Eddleston, M., et al (2006) Deaths from pesticide poisoning: a global response. British Journal of Psychiatry, $\mathbf{1 8 9}$, 201-203.
Joseph, A., Abraham, S., Muliyil, J. P., et al (2003) Evaluation of suicide rates in rural India using verbal autopsies, 1994-99. BMJ, 326, |I2I-II22.

Prasad, J., Abraham, V. J., Minz, S., et al (2006) Rates and factors associated with suicide in Kaniyambadi Block, Tamil Nadu, South India, 2000-02. International Journal of Social Psychiatry, 52, 65-7I.

R. Alex, J. Prasad, A. Kuruvilla, K. S. Jacob Christian Medical College, Vellore 632002, India. Email: sanju@cmcvellore.ac.in doi: 10.1192/bjp.190.3.274a

\section{One hundred years ago}

\section{The increase of temperance}

The Inland Revenue returns show a steadily progressive decrease in the consumption of beer and spirits in the United Kingdom since 1899; that is in encouraging contrast with the equally steady but more rapid increase up to that date.

The beer consumption in 1899-1900 was 32.2 gallons per head of the population, making a total of 36.5 million barrels, but in 1905-1906 this had fallen to 27.9 gallons per head and to 33.5 million barrels.

The spirit consumption has also fallen each year from 1.17 gallons per head and a total of 48 million gallons for 1889-
1900 to .90 gallons per head and 39.1 million gallons in 1905-1906.

The reduction in the consumption of spirits is very striking, and in addition to the reduction in the total quantity of beer consumed there is to be added the large increase in the proportion of the lighter beers of home and foreign manufacture.

Pauperism, crime, and insanity are so largely attributable to the abuse of alcoholic drinks that the statistics of each should be carefully watched during the next few years for any indication of an improvement. It is, of course, possible that this reduction may be due only to the greater moderation from necessity or improved habits of the middle and upper classes only, although it would appear to be too large to be thus explained.

Abuse of alcohol, in the statistics of the causes of insanity, has fluctuated very little for many years past, so that any distinct diminution would be very significant, and should encourage a still more vigorous crusade in favour of true temperance - the use without abuse of the cup that cheers and may inebriate.

\section{REFERENCE}

Journal of Mental Science, January 1907, 146.

Researched by Henry Rollin, Emeritus Consultant Psychiatrist, Horton Hospital, Epsom, Surrey doi: 10.I192/bjp.190.3.275 